\title{
Image Transmission in Military Network Using Bézier Curve
}

\author{
Syamsul Rizal and Dong-Seong Kim
}

\begin{abstract}
This paper proposed a method for reconstruct image by using Bézier Curve function. One of the causes of errors in image reconstruction is lack of sampling. In order to solve that problem, this paper proposes a method by using Bézier Curve to interpolate data between sampling points to reduce the error. The proposed method is compared to the previous works; compressive sampling and discrete Fourier transform (DFT). Compressive sampling is a method which has small number of data sample and DFT is used to perform Fourier analysis in many practical applications in digital signal processing. The result shows that image reconstruction using Bézier curve has closer quality to the original image compared to compressive sampling and DFT while it can maintain small size of reconstructed images.
\end{abstract}

Index Terms-Bézier curve, compressive sampling, DFT, Image reconstruction.

\section{INTRODUCTION}

Distributed system is a single set of computer connected by a computer networks and equipped with distributed system software. The performance of the distributed system is affected by network-induced parameters such as delays, jitters, packet losses and link failures [1], [2]. This system has heavy application, such as those in the fields of aerospace, military visual processing, or industrial computer processors [3]. Due to large variation of data size, signal processing was needed to reconstruct the data. Image processing is one form of signal processing where the input is an image, such as photographs or video frame, while the output of image processing can be either an image or a set of characteristics or parameters related to the image. The reconstruction process of an image from the acquired data is an inverse problem. Often, it is impossible to exactly solve the problem inversely. Though the image is reconstructed, the result is far from the original image. Many famous techniques are used in image processing such as compressive sampling, discrete Fourier transform (DFT), etc.

Compressive sampling or compressed sensing (CS) is a novel sampling/sensing paradigm that goes against the common method in data acquisition. In CS, system can recover certain signals by using fewer samples compared to traditional method such as Nyquist theorem, where the sampling data must be at least twice the maximum frequency used in the signal [4]. Compressive sampling is commonly used in image or video processing due to its small data

Manuscript received September 10, 2014; revised March 31, 2015.

The authors are with the School of Electronic Engineering, Kumoh National Institute of Technology, South Korea (e-mail: syamsul@kumoh.ac.kr,dskim@kumoh.ac.kr). compression. The study in [5], [6] demonstrated how compressing sampling method used in image restoration. Sampling scale from 10 to 60 percent are compared. The results showed that received images are reconstruct-able and readable by using small sampling scale.

Besides compressive sampling, Discrete Fourier Transform (DFT) is used for image processing. DFT is used to transform original image from spatial to frequency domain [7]-[9]. In image processing, the samples can be the values of pixels along a row or column of an image.

This paper proposed a technique to reconstruct the image using Bézier curve function to interpolate the data between each sampling point. Bézier curve is a set of independent variables that represent the coordinates of several points in curved line between two points. Hence, the data between sampling points can be predicted and reconstructed using this method. Using image processing technique, the image size will become smaller than the original image with good quality. These techniques can increases energy efficient and send the image faster than the original due to the small size of the image [10].

The remain of this paper is organized as follows: Section II describes the compressive sampling and DFT, Section III explains the proposed method. Results and analysis are explained in Section IV. Finally, Section V concludes the paper.

\section{IMAGE PROCESSING TECHNIQUES}

Image processing is a technique to compressed and reconstructed the image to become the image size was smaller than the original image but with the image that resembles the actual image. Many techniques can be used to reconstructed image in image processing, such as compressive sampling and DFT.

\section{A. Compressive Sampling Overview}

Compressive sampling is a compression method with a random collection an amount of data which is determined by the value of the rate measurement. This method used 2 transformations; sparsity and incoherent transform [10], [11]. A sensing mechanism in which information about signal $f(t)$ is obtained by linear functional recording this value.

$$
y_{k}=\left\langle f, \psi_{k}\right\rangle k=1, \ldots, m
$$

That correlates the object that we wish to acquire with the waveforms $\psi_{k}(t), y$ is a vector of sampled values of $f$ in the time or space domain, Dirac delta functions (spikes) are sensing waveform. If the sensing waveforms are indicator 
functions of pixels, then $y$ is the image data typically collected by sensors in a digital camera. If the sensing waveforms are sinusoids, then $y$ is a vector of Fourier coefficients.

\section{1) Sparsity}

Sparsity expresses the idea that the information rate of a continuous signal may be much smaller than suggested by its bandwidth. Mathematically, a vector $f \in \mathbf{R}^{n}$ (such as the $n$-data) which expanded in orthonormal basis (such as a wavelet basis) $\Psi=\left[\psi_{1}, \psi_{2}, \ldots, \psi_{n}\right]$ as follows:

$$
f(t)=\sum_{i=1}^{n} x_{i} \psi_{i}(t)
$$

where $x$ is the coefficient sequence of $f, x_{i}=\left(f, \psi_{i}\right)$. It will be convenient to express $f$ as $\psi_{x}$, where $\Psi$ is the $n \times n$ matrix with $\psi_{1}, \ldots, \psi_{n}$ as columns. The implication of sparsity is now clear: when a signal has a sparse expansion, one can discard the small coefficients without much perceptual loss.

\section{2) Incoherence}

Incoherence extends the duality between time and frequency and expressed the idea that objects having a sparse representation in $\Psi$ must be spread out in the domain in which they are required. Suppose there is a pair $(\Phi, \Psi)$ of orthobases of $\mathbf{R}^{n}$. The first basis $\Phi$ is used for sensing the object $f$ as in (1) and the second is used to represent $f$. The coherence between the sensing basis $\Phi$ and the representation basis $\Psi$ is

$$
\mu(\Phi, \Psi)=\sqrt{n} \max _{1 \leq k, j \leq n}\left|\left\langle\varphi_{k}, \psi_{j}\right\rangle\right|
$$

The coherence measures the target correlation between any two elements of $\Phi$ and $\Psi$. If $\Phi$ and $\Psi$ contain correlated elements, the coherence is large. Otherwise, it is small. As for how large or small, it follows from linear algebra that $\mu(\Phi, \Psi)=[1, \sqrt{n}]$.

Yet, compressive sampling needs longer time reconstruct the image due to its fewer sampling method.

\section{B. Fourier Transform Overview}

In mathematics, the discrete Fourier transform (DFT) is one of the specific forms of Fourier analysis. It transforms one function to another, which is called the frequency domain representation of the time domain. Yet the DFT requires an input function that is discrete and whose non-zero values have a limited (finite) duration.

Since the input function is a finite sequence of real or complex numbers, the DFT is ideal for processing information stored in computers. In particular, the DFT is widely employed in signal processing and related fields to analyze the frequencies contained in a sampled signal, to solve partial differential equations, and to perform other operations such as convolutions. The DFT can be computed efficiently in practice using a fast Fourier transform (FFT) algorithm.

FFT algorithms are so commonly employed to compute the DFT, the two terms are often used interchangeably in colloquial settings, although there is a clear distinction: "DFT" refers to a mathematical transformation, regardless of how it is computed, while "FFT" refers to any one of several efficient algorithms for the DFT.

The sequence of $N$ complex numbers $x_{0}, \ldots, x_{N-1}$ is transformed into the sequence on $N$ complex numbers $x_{0}, \ldots$, $x_{n-1}$ by the DFT according to the formula: where is a primitive $N^{\text {th }}$ root of unity.

\section{Bezier Curve}

Bézier curve is a set of independent variables that represent the coordinates of several points in curved line between two or more points. Bézier curve is a common method to smoothing any image of curve based on its points or degrees.

The study in [12] presented how Bézier curve is used for enhance and transform the pixel intensity for increase image depth for any display including computer, mobile phone, or others. In [13] and [14] presented how Bézier curve generate shape of image then modified it according to its points and degrees. Bézier curve also used for smoothing hand-writing in electronic method to make smoother [15].

\section{SYSTEM MODEL}

Data frame structure is a basic method to describe this proposed method. Fig. 1 shows compressive sampling and proposed method data frame structure. The data frame is consisted of data and protector part. Protector parts usually represented as the error correction such as CRC, ECC, etc. This paper proposed a progressive data transmission using Bézier curve formula, which is described in Fig. 1.

a)

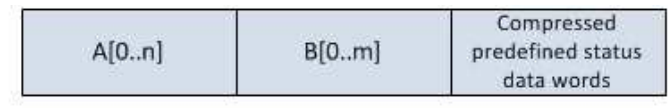

b)

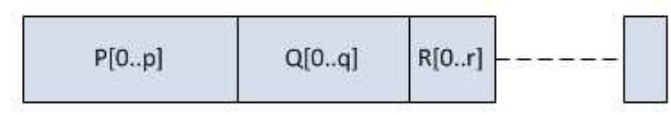

Fig. 1. The data frame structure. a) Compressive data transmission. b) Functional data transmission.

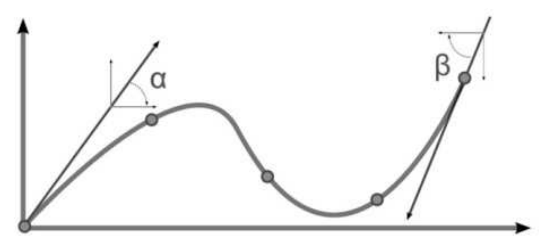

Fig. 2. Cubic Bézier curve.

\section{A. Bézier Curve}

A Bézier curve is defined by a set of control points $\mathrm{P}_{0}$ through $P_{n}$, where $n$ is Bézier order ( $n=1$ for linear, 2 for quadratic, etc.). The first and last control points are always the end points of the curve. However, the intermediate control points generally do not lie on the curve.

Consider the following cubic-Bézier formulation. Define $A$ is the set of number where $a_{0}=|A|_{\min }$ and $a_{n-1}=|A|_{\max }$. Therefore, there exist such a $P$ that represents $A$ where $|P| \leq|A|$ and $A \in C$.

In this paper we consider the cubic- Bézier where the curve is represented by 4 points. Fig. 2 shows the possibilities to cover a data which consists of 5 numbers data that lies on the curve within a set that can be represented 4 numbers of cubic- 
Bézier. It means that by sending 4 data from Bézier function, we can construct 5 data on the receiver.

The extended version of the cubic Bézier, double cubic Bézier, where 6 numbers of Bézier function, can be used to represent 9 numbers of data. This process can be described as the subdividing process of the curve. In other words, every curve can be scooped within the Bézier function depends on the depth of the curve. Therefore, the points of one Bézier curve can be extended as follows,

$$
p_{i}^{j}=(1-\tau) p_{i}^{j-1}+\tau p_{i+1}^{j-1}
$$

Eq. (4) shows that every data can be approached by any Bézier curved. The detail level is formulated corresponds directly with the level of Bézier curve division.

\section{B. Proposed Method}

\section{1) Transmitter}

When the transmitter wants to send image, the image must be converted into matrix with size that transmitter and receiver agreed. Let matrix $A$ with size $B \times B$, where $B$ is divisible by 5 . The value of matrix $A$ is the depth of the color of each pixel of the image from 0 to 255 for black and white image as shown in Fig. 3. Then this matrix is transformed by separating each 5 columns into 1 new column with to be new matrix $A$ with size $5 \times K$ where $K$ is the result of $B$ div 5 . Note that though Bézier curve can be used to approximate more than 5 points; we use the minimum approximation to get the best result.

$$
A=\left[\begin{array}{cccc}
A_{11} & A_{12} & \cdots & A_{1 B} \\
A_{21} & A_{22} & \cdots & A_{2 B} \\
\vdots & \vdots & \vdots & \vdots \\
A_{B 1} & A_{B 2} & \cdots & A_{B B}
\end{array}\right]
$$

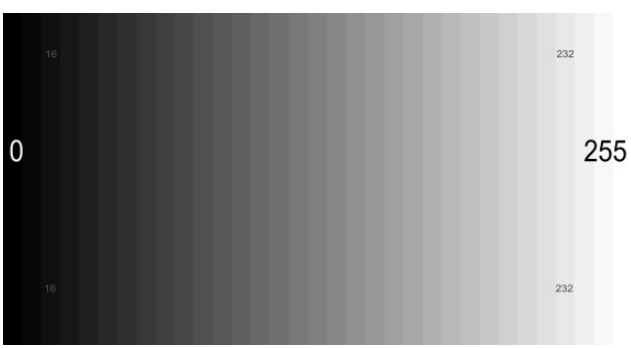

Fig. 3. Grayscale image value.

Then we reproduce matrix $A$ so that

$$
A=\left[\begin{array}{cccc}
A_{11} & A_{12} & \cdots & A_{1 K} \\
A_{21} & A_{22} & \cdots & A_{2 K} \\
A_{31} & A_{32} & \cdots & A_{3 K} \\
A_{41} & A_{42} & \cdots & A_{4 K} \\
A_{51} & A_{52} & \cdots & A_{5 K}
\end{array}\right]
$$

where $A_{11}$ has equal value to $A_{11}, A_{12}$ is equal to $A_{61}$, and so until $A_{5 K}$ is equal to $A_{B B}$. Then for each column the 5 values are approximated to get four Bézier control points $P$.

$$
A=\left[\begin{array}{cccc}
P_{11} & P_{12} & \cdots & P_{1 K} \\
P_{21} & P_{22} & \cdots & P_{2 K} \\
P_{31} & P_{32} & \cdots & P_{3 K} \\
P_{41} & P_{42} & \cdots & P_{4 K}
\end{array}\right]
$$

From the current matrix $A$ the number of Bézier value that must be reconstructed is equal to $K$. Then the transmitter transmit matrix $A$ in one row matrix.

$$
A=\left[\begin{array}{llllll}
P_{11} & P_{21} & \cdots & P_{1 K} & \cdots & P_{4 K}
\end{array}\right]
$$

\begin{tabular}{l} 
Algorithm 1: Transmitter \\
\hline 1: Set image $(A)$ \\
2: Size $(A)=B \times B ; B \bmod 5=0$. \\
3: A $=$ Reshape $(A)$ \\
4: Size $(A)=5 \times K$ \\
5: $i=0$ \\
6: for $i<K ; \mathrm{i}++$ do \\
7: Read column $(i)$ \\
8: $\quad$ Get Bezier point for $Q[i]$ \\
9: end for \\
10: Size $(A)=P \times K ; P=4$ \\
11: A $=\operatorname{Reshape~}(A)$ \\
12: Size $(A)=1 \times Q ; Q=4 \times K$ \\
13: Send $A$ \\
\hline
\end{tabular}

1

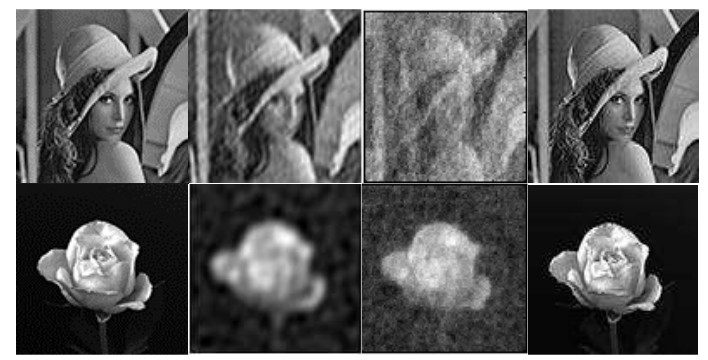

3

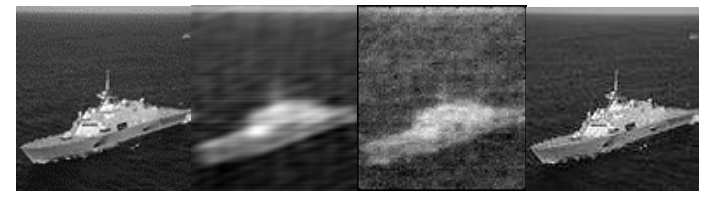

a

b

$\mathrm{c}$

d

Fig. 4. Image reconstruction: (a). Original. (b). DFT. (c). Compressive Sampling. (d). Bezier curve.

Receiver, Let the receiver has converted image, then for every $4 P$ points of data $P_{1}, P_{2}, P_{3}$, and $P_{4}$. Bézier curve is applied to interpolate the data. Let $P_{1}$ and $P_{4}$ are the start and the end point of Bézier curve, $P_{2}$ and $P_{3}$ are intermediate points which are used as control points to create the Bézier curve, Fig. 2. This algorithm is repeated until the Bézier curve covers all $\mathrm{P}$ received data. After Bézier curve is obtained, the receiver extracts its function and reconstructs the data based on Bézier curve. Algorithm 2 explained more detail about how to reconstruct image. 


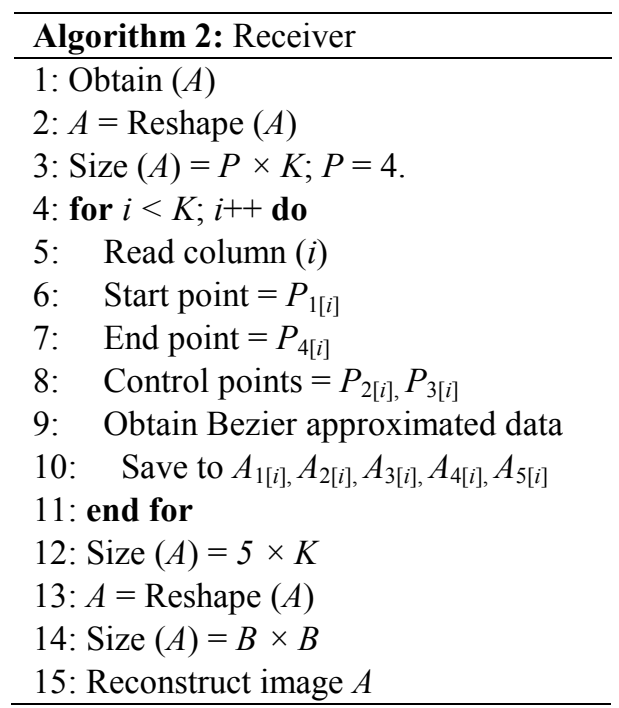

\section{RESULT AND ANALYSIS}

In this simulation, we consider image $100 \times 100$ pixels square to be reconstructed. We compare the reconstructed image by using compressive sampling, DFT, and Bézier curve. We use standard DFT and 10 percent of data sampling in compressive sampling. Black and white images are used in the simulation to make ease, faster calculation and reconstruction.

Fig. 4 shows 3 simulation results of image reconstruction. Fig. 4(a) is the original images which are reconstructed. In both Fig. 4(b) and Fig. 4(c) show that the images cannot be reconstructed well. The qualities of the images are poor. In Fig. 4(d) the quality of image are better than the other methods (CS and DFT).

In order to make certain that the proposed method has better image reconstruction the pixel value similarity is calculated. Table I shows the exact similarity of each pixel in each reconstruction method compared to the original images. Bézier curve reconstruction method has $12.34 \%, 33.78 \%$ and $12.52 \%$ pixel similarity of image 1,2 and 3 respectively. While DFT is only $1.38 \%, 1.40 \%$, and $3.31 \%$, and CS are very poor pixel similarity with only around $1 \%$.

\begin{tabular}{cccc}
\multicolumn{4}{c}{ TABLE I: PIXEL SIMILARITY } \\
\hline \hline Image & DFT & CS & Bezier Curve \\
\hline 1 & $1.38 \%$ & $0.85 \%$ & $12.34 \%$ \\
2 & $1.40 \%$ & $0.29 \%$ & $33.78 \%$ \\
3 & $3.31 \%$ & $1.16 \%$ & $12.52 \%$ \\
\hline \hline
\end{tabular}

Therefore, Bézier curve has more than $10 \%$ pixel similarity of each image. Since the grayscale image has 256 values, we calculate the image similarity. We compute the difference level of each pixel compare to the original image. $4 \%, 10 \%$, and $20 \%$ mean the difference value of reconstructed images and original images are less than 10 , less than 25 , and less than 50 respectively.

TABLE II: IMAGE SIMILARITY IN BEZIER CURVE RECONSTRUCTION

\begin{tabular}{cccc}
\hline \multicolumn{4}{c}{ Grayscale difference level } \\
\hline Image & $4 \%$ & $10 \%$ & $20 \%$ \\
\hline 1 & $94.84 \%$ & $99.81 \%$ & $100 \%$ \\
2 & $96.26 \%$ & $99.67 \%$ & $100 \%$ \\
3 & $96.06 \%$ & $99.60 \%$ & $100 \%$ \\
\hline \hline
\end{tabular}

Table II and Fig. 5 show that with the $4 \%$ difference level the reconstructed images have $95 \%$ similarity. The $10 \%$ difference level the reconstructed images has $99 \%$ similarity, and in $20 \%$ difference level the reconstructed images has $100 \%$ similarity. It means that each pixel value of reconstructed images is very close to the original images pixel value.

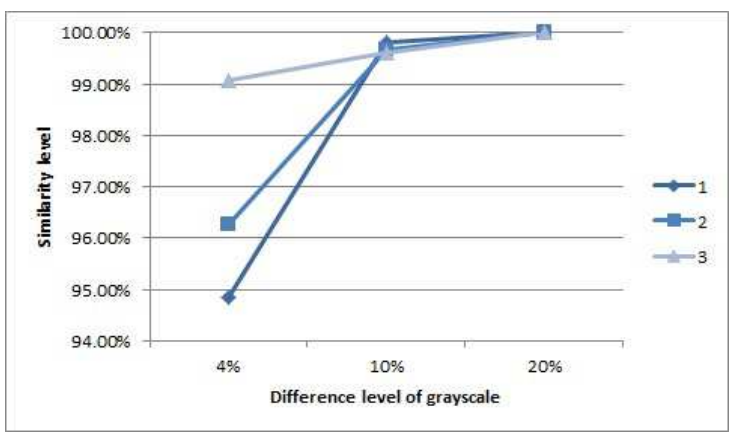

Fig. 5. Similarity level of Bezier curve reconstructed images.

Beside the image quality, compression method is useful for reduce the size of image. Table III shows image size of each method. The image size from the proposed method is much smaller than compressive sampling and DFT methods or even the original image. By using the proposed method we can reduce the image size smaller without harm its quality.

\begin{tabular}{ccccc}
\multicolumn{5}{c}{ TABLE III: IMAGE SIZE (IN KB) } \\
\hline \hline Image & Original & DFT & CS & Bezier Curve \\
\hline 1 & 16.6 & 5.69 & 9.77 & 3.08 \\
2 & 12.3 & 3.64 & 8.71 & 1.71 \\
3 & 22.3 & 17.8 & 30.6 & 2.00 \\
\hline \hline
\end{tabular}

Another image size has been simulated using Bézier curve function. Table IV shows the image size ratio before send the image to the receiver. The image size for this simulation is $142 \mathrm{~KB}$ with $1296 \times 964$ pixels for image dimension. The image size ratio is the ratio between the original image sizes with constructed image size before it is sent to receiver. The biggest ratio is 4.0; it is for simulation number 5 and 9 . It means the constructed image size is smaller than the others. However, the pixel similarity is the worst in these simulations (number 5 and 9). It is the trade off in this proposed method. The bigger the value of image size ratio, the smaller the percentage of pixel similarity, and vice versa. Fig. 6 is the graph for pixel similarity. The 0 values mean that this paper did not make a simulation for some cases.

TABLE IV: IMAge Size RATIO Vs PiXel Similarity

\begin{tabular}{cccccc}
\multicolumn{7}{c}{ TABLE IV: IMAGE SIZE RATIO VS PIXEL SIMILARITY } \\
\hline \hline No & $\begin{array}{c}\text { Number } \\
\text { of Data }\end{array}$ & $\begin{array}{c}\text { Number } \\
\text { Control } \\
\text { Points }\end{array}$ & $\begin{array}{c}\text { Image Size Before } \\
\text { Transmission } \\
(\mathrm{KB})\end{array}$ & $\begin{array}{c}\text { Image } \\
\text { Size } \\
\text { Ratio }\end{array}$ & $\begin{array}{c}\text { Pixel } \\
\text { Similarity } \\
(\%)\end{array}$ \\
\hline 1 & 9 & 8 & 126.18 & 1.13 & 69.004 \\
2 & 10 & 8 & 113.56 & 1.25 & 69.825 \\
3 & 10 & 9 & 127.76 & 1.11 & 70.203 \\
4 & 12 & 9 & 106.46 & 1.33 & 68.216 \\
5 & 16 & 4 & 35.49 & 4.00 & 64.265 \\
6 & 16 & 6 & 53.23 & 2.67 & 65.765 \\
7 & 16 & 8 & 70.97 & 2.00 & 66.665 \\
8 & 16 & 9 & 79.85 & 1.78 & 67.139 \\
9 & 24 & 6 & 35.49 & 4.00 & 63.767 \\
10 & 24 & 8 & 47.32 & 3.00 & 64.895 \\
11 & 24 & 9 & 53.23 & 2.67 & 65.260 \\
\hline \hline
\end{tabular}




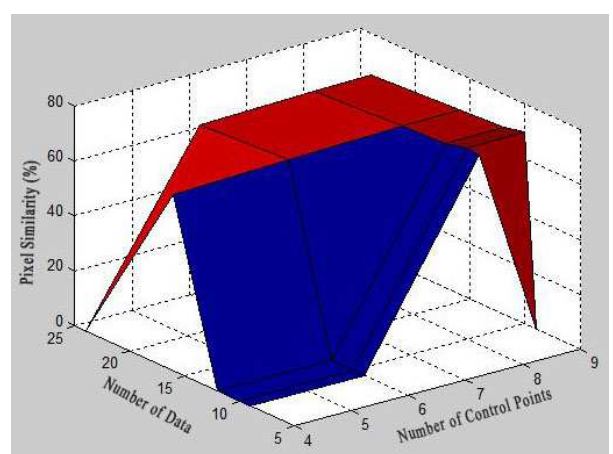

Fig. 6. Pixel similarity in 3D graph.

Table $\mathrm{V}$ shows the processing time for each method in different simulation tools. This paper use MATLAB and OpenCV for comparison the time to reconstruct the image. This paper used 2 operating systems to make simulation. Windows 7 64-bit is used for MATLAB and Ubuntu 13.04 64-bit is used for OpenCV. The processing time is calculated in these simulations. The processing time for Bézier curve have smaller number than the other methods. Image dimension $100 \times 100$ pixels has 0.8 and 0.02 second for MATLAB and OpenCV respectively when using Bézier curve. The other methods need more than 40 second to reconstruct $100 \times 100$ pixels image when using MATLAB as simulation tools. The processing time for $1296 \times 964$ pixels, the Bézier curve still have the smallest number. When using OpenCV, the Bézier curve need 0.4 second to reconstruct the image. However, the processing time needed for MATLAB almost 18 times, around 7.5 second.

TABLE V: PROCESSING TIME (SECOND)

\begin{tabular}{lcccccc}
\hline \hline Image & \multicolumn{4}{c}{ MATLAB } & \multicolumn{3}{c}{ OpenCV } \\
\cline { 2 - 7 }$($ Pixels) & DFT & CS & BC & DFT & CS & BC \\
\hline $100 \times 100$ & 42 & 57 & 0.8 & 0.62 & 2.3 & 0.02 \\
$1296 \times 964$ & 329 & 402 & 7.5 & 2.54 & 11.4 & 0.4 \\
\hline \hline
\end{tabular}

\section{CONCLUSIONS}

In this paper, an approach to reconstruct data by taking advantage of Bezier curve was presented. Compressive sampling is a sampling method that against sampling paradigm that the number of sampling is much less than the original data. This method caused data error in reconstruction. DFT is a method which is used commonly in image processing. This paper presented a method to help image reconstruction by adding a function called Bezier curve to interpolate data between sampling points the reconstruct data.

The cubic Bezier curve is used in this paper; this method takes 4 sampling points to interpolate and reconstruct 5 values of the image $(100 \times 100$ pixels $)$. By using this method, the reconstructed image has better quality and higher similarity level compared to the other image reconstruction methods.

\section{ACKNOWLEDGMENT}

This research was supported by the MSIP (Ministry of Science, ICT and Future Planning), Korea, under the "Creative ICT Convergence Human Resource Development Program" support program supervised by the NIPA (National IT Industry Promotion Agency).

\section{REFERENCES}

[1] D.-S. Kim, D. H. Choi, and P. Mohapatra, "Real-time scheduling method of discrete networked control systems," Control Engineering Practice, vol. 17, no. 5, pp. 654-570, May 2009.

[2] D.-S. Kim, Y. S. Lee, W. H. Kwon, and H. S. Park, "Maximum allowable delay bounds of networked control systems," Control Engineering Practice, vol. 11, no. 11, pp. 1301-1313, 2003.

[3] A. Prasetiadi and D.-S. Kim, "Faulty Node Detection in Distributed Systems Using BCH Code," IEEE Communications Letters, vol. 17, no. 3, pp. 620-623, March 2013.

[4] E. Candes and M. Wakin, "An introduction to compressive sampling," IEEE Signal Processing Magazine, vol. 25, no. 2, pp. 21-30, 2008.

[5] J. Wormann, S. Hawe, and M. Kleinsteuber, "Analysis based blind compressive sensing," IEEE Signal Processing Letters, vol. 20, no. 5, pp. 491-494, May 2013.

[6] A. Wani and N. Rahnavard, "Compressive Sampling for energy efficient and loss resilient camera sensor networks," in Proc. Military Communications Conference, 2011, pp. 1766-1771.

[7] J. Mandal and A. Khamrui, "A genetic algorithm based steganography in frequency domain (GASFD)," in Proc. International Conference on Communication and Industrial Application, 2011, pp. 1-4.

[8] B. Varma, K. Paul, and M. Balakrishnan, "Accelerating 3D-FFT using hard embedded blocks in FPGAs," in Proc. 26th International Conference on VLSI Design and 12th International Conference on Embedded Systems, January 2013, pp. 92-97.

[9] G. Rath and C. Guillemot, "Performance analysis and recursive syndrome decoding of DFT codes for bursty erasure recovery," IEEE Transactions on Signal Processing, vol. 51, no. 5, pp. 1335-1350, 2003.

[10] F. Chen, F. Lim, O. Abari, A. Chandrakasan, and V. Stojanovic, "Energy Aware Design of Compressed Sensing Systems for Wireless Sensors Under Performance and Reliability Constraints," in Proc. IEEE Transactions on Circuits and Systems I: Regular Papers, vol. 60, no. 3, pp. 650-661, March 2013.

[11] X. Wang, Z. Zhao, N. Zhao, and H. Zhang, "On the application of compressed sensing in communication networks," in Proc. 5th International ICST Conference on Communications and Networking in China (CHINACOM), August 2010, pp. 1-7.

[12] F.-C. Cheng and S.-C. Huang, "Efficient histogram modification using bilateral bezier curve for the contrast enhancement," Journal of Display Technology, vol. 9, no. 1, pp. 44-50, 2013.

[13] T. Gangdou and W. Ke, "Shape control and modification of rational bezier curve and surface," Journal of Systems Engineering and Electronics, vol. 2, no. 2, pp. 65-72, 1991.

[14] F. Sohel, G. Karmakar, L. Dooley, and M. Bennamoun, "Bezier curvebased generic shape encoder," IET Image Processing, vol. 4, no. 2, pp. 92-102, 2010.

[15] H. Cao and A. Kot, "Lossless data embedding in electronic inks," IEEE Transactions on Information Forensics and Security, vol. 5, no. 2, pp. 314-323, 2010.

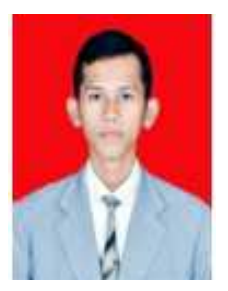

Syamsul Rizal was born in West Java Province, Indonesia, in 1988. He received the B.S. degree from the Telkom Institute of Technology Bandung, Indonesia, in 2011. He is currently pursuing the M.S. degree in IT Convergece Department, Kumoh National Institute of Technology (KIT), South Korea. His research interests include military standard, industrial informatics, coding decoding, signal processing and information theory.

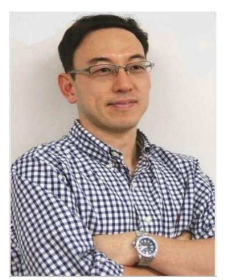

Dong-Seong Kim received his Ph.D. degree in electrical and computer engineering from the Seoul National University, Seoul, Korea, in 2003. From 1994 to 2003 , he worked as a full-time researcher in ERC-ACI at Seoul National University, Seoul, Korea. From March 2003 to February 2005, he worked as a postdoctoral researcher and visiting scholar at the Wireless Network Laboratory in the School of Electrical and Computer Engineering at Cornell University, NY. He was a visiting professor with Department of Computer Science, University of California, Davis, U.S.A. Since 2004, he has been a professor in the School of Electronic Engineering, a director of ICT-CRC and mobile research center at Kumoh National Institute of Technology, Korea. His current main research interests are industrial wireless control network, networked embedded system, and fieldbus. 